\title{
Systemic CD4+ and CD8+ T-cell cytokine profiles correlate with GOLD stage in stable COPD
}

Marthe S. Paats, Ingrid M. Bergen, Henk C. Hoogsteden, Menno M. van der Eerden and Rudi W. Hendriks

ABSTRACT: Chronic obstructive pulmonary disease (COPD) is associated with pulmonary and systemic inflammation. Both CD4+ and CD8+ T-lymphocytes play a key role in COPD pathogenesis, but cytokine profiles in circulating T-lymphocytes have not been well characterised.

Here we report the analysis of peripheral blood T-cells from 30 stable COPD patients and 10 healthy never-smokers for interferon (IFN)- $\gamma$, interleukin (IL)-4, tumour necrosis factor (TNF)- $\alpha$ and the T-helper 17 cytokines IL-17A, IL-17F and IL-22 by intracellular flow cytometry.

We found significantly increased proportions of IFN- $\gamma+$ and TNF- $\alpha+$ CD8+ T-cells in COPD patients, when compared with healthy controls. This was most evident in patients with less severe disease. In contrast, expression profiles in circulating CD4+ T-cells were similar in COPD patients and healthy controls for all cytokines tested, except for IL-17F. COPD patients with more severely reduced diffusing capacity had lower proportions of IL-17A+ CD4+ T-cells. Proportions of IL-22+ cells in the CD4+ memory T-cell population were significantly increased in active smokers, when compared with past smokers.

Collectively, this comprehensive cytokine analysis of circulating T-cells in COPD patients revealed a correlation for CD8+ T-cells between Global Initiative for Chronic Obstructive Lung Disease (GOLD) stage and IFN- $\gamma$ or TNF- $\alpha$ expression, but not for CD4+ T-cells.

KEYWORDS: Chronic obstructive pulmonary disease, cytokine, flow cytometry, T-helper 17, T-lymphocytes

hronic obstructive pulmonary disease (COPD) is a major cause of morbidity and mortality throughout the world [1]. Tobacco smoking is the primary risk factor but other noxious particles can also cause a poorly reversible airflow limitation, which is the principal feature of COPD. Chronic inhalation of toxic particles and gases causes destruction of lung parenchyma, activates epithelial cells, increases mucus production and stimulates migration of many inflammatory cells $[2,3]$. This results in an abnormal inflammatory response in the small airways and alveoli in which many inflammatory cells are involved, including neutrophils, macrophages and T-lymphocytes [3, 4].

Different T-cell subsets are identified in the pathogenesis of COPD. CD8+ T-cells predominate over CD4+ T-cells in the airways and lung parenchyma [5]. Several reports have shown that increased bronchus obstruction correlates with increased interferon (IFN)- $\gamma$ production by lung
CD8+ T-cells [5-7] and their increased expression of cytotoxic molecules [8]; however, interleukin (IL)-4 production has also been reported [9]. CD4+ T-cells that accumulate in the airways and lungs of patients also mainly produce IFN- $\gamma$ and therefore have a T-helper 1 (Th1) phenotype [10]. However, recent studies have shown increased expression of Th17 cytokines in bronchial mucosa and sputum of patients, indicating a role for this novel T-helper subtype in COPD [11-13].

Besides airway inflammation, the presence of systemic inflammation has been suggested by multiple studies showing increased oxidative stress, the activation of circulating neutrophils and lymphocytes, and altered circulating levels of inflammatory mediators and acute phase proteins including TNF- $\alpha$ and its receptors, IL- 6 and IL- 8 , and Creactive protein [14-16]. Nevertheless, the cytokine profile of peripheral blood T-cells from COPD patients has not been as well characterised as that from T-cells in the lung. To our knowledge,
AFFILIATIONS

Dept of Pulmonary Medicine, Erasmus MC Rotterdam, Rotterdam, The Netherlands.

CORRESPONDENCE M.S. Paats Dept of Pulmonary Medicine Erasmus MC Rotterdam P0 Box 2040 NL 3000 CA Rotterdam The Netherlands

E-mail: m.paats@erasmusmc.nl

Received:

May 112011

Accepted after revision:

Nov 272011

First published online:

Dec 192011 
the Th17 subset in the peripheral blood of stable COPD patients has not yet been studied.

The aim of this study was to determine intracellular cytokine profiles of circulating T-cells in patients with stable COPD, analysing both CD4+ and CD8+ T-cells, thereby providing a detailed characterisation of a wide variety of cytokines, including those produced by the Th1, Th2 and novel Th17 subsets. We correlated the cytokine profiles with Global Initiative for Chronic Obstructive Lung Disease (GOLD) classification, lung function parameters and smoking status.

\section{METHODS}

\section{Subjects}

The study population consisted of 30 stable COPD patients (forced expiratory volume in $1 \mathrm{~s}$ (FEV1)/forced vital capacity $(\mathrm{FVC})<70 \%$ ) and 10 healthy individuals with normal lung function and no history of pulmonary disease and smoking. All subjects had no known autoimmune disease, atopic diseases or allergies. In addition, none of the COPD patients had chronic bacterial colonisation of the airways or recurrent lower respiratory tract infections (four or more infections per year) as defined by the Dutch national guidelines [17]. COPD patients did not have an exacerbation episode in the 4 weeks prior to inclusion. They were all treated with inhaled bronchodilatators and inhaled corticosteroids (ICS), but did not receive oral steroid therapy or antibiotic treatment. ICS doses ranged from $50 \mu \mathrm{g}$ once a day to $400 \mu \mathrm{g}$ four times daily. Patients were categorised into GOLD stages II-IV based on pulmonary function tests $[18,19]$. Past smokers were defined as not having smoked in the previous year. Peripheral blood was drawn from all subjects after written informed consent. The study was approved by the medical ethical committee of the Erasmus Medical Centre Rotterdam, Rotterdam, the Netherlands.

\section{Pulmonary function tests}

All subjects underwent pulmonary function testing according to the 2005 American Thoracic Society (ATS) spirometry standards [20]. FVC and FEV1 were measured to determine airflow limitation (FEV1/FVC $<70 \%$; FEV1 $<80 \%$ of predicted value). Diffusing capacity of the lung for carbon monoxide $(T \mathrm{~L}, \mathrm{CO})$ and the transfer coefficient of the lung for carbon monoxide corrected for the alveolar volume (KCOc) were also determined. Diffusing capacity parameters were expressed as percentages of reference values.

\section{Cell isolation from peripheral blood and flow cytometry analysis}

Peripheral blood mononuclear cells (PBMC) were isolated using standard Ficoll-Paque (GE Healthcare, Uppsala, Sweden) density gradient and resuspended in IMDM medium (Iscove's Modified Dulbecco's Media) (BioWhittaker, Verviers, Belgium) containing $10 \%$ fetal calf serum, $\beta$-mercaptoethanol and gentamycin. For cytokine analysis, cells were stimulated with ionomycine (Sigma, Zwijndrecht, the Netherlands) and phorbol myristate acetate (Sigma) in the presence of Brefeldin A (eBiosciences, Vienna, Austria) and incubated at $37^{\circ} \mathrm{C}$. After $4 \mathrm{~h}$ cells were collected, centrifuged for $7 \mathrm{~min}$ at $400 \times g$, resuspended in fluorescence-activated cell sorter buffer (PBS supplemented with $0.25 \%$ bovine serum albumin and 0.5 $\mathrm{mM}$ EDTA and $0.05 \% \mathrm{NaN}_{3}$ ) and stained with conjugated monoclonal antibodies to detect membrane markers. To detect intracellular cytokines, cells were subsequently fixed with $2 \%$ paraformaldehyde (PFA) fixation (Merck, Amsterdam, the Netherlands) and permeabilised with $0.5 \%$ saponin solution (Sigma). For intracellular FoxP3 detection the FoxP3 Staining Buffer Set (eBiosciences) was used. The following antibodies were applied: anti-CD3 antigen-presenting cell (APC)-AF780, anti-CD8 APC, anti-CD45RO eFluor 650NC, anti-IL-17A AF647, anti-IL-17F phycoerythrin (PE), anti-IFN- $\gamma$ PerCPCy5.5, anti-TNF- $\alpha$ PerCP-Cy5.5, anti-IL-4 Pe-Cy7, anti-FoxP3 APC (all eBiosciences), anti-CD4 fluorescein isothiocyanate, anti-CD45RO PE, anti-CD25 Pe-Cy7 (all BD Biosciences, Breda, the Netherlands), anti-CD45RA PE-TxR (Invitrogen, Bleiswijk, the Netherlands) and anti-IL-22 APC (R\&D Systems, Abingdon, UK). Cells were measured on a LSRII Flowcytometer (BD Biosciences); data were analysed using FlowJo (BD) software. Isotype controls and fluorescence minus one controls were used to set gates. To present the data we used proportions of cells. The absolute number of the cells depends on the number of gated events and on absolute numbers of lymphocytes. To present the proportion is more common in literature and seems to be more objective in comparative studies. Two separate experiments were performed using the same subject samples showing very similar results.

\section{Statistical analysis}

Parametric data were expressed as mean values with $95 \%$ confidence interval; nonparametric data were described as median values with interquartile range. Differences between two groups were evaluated by using the Mann-Whitney Utest. Across-group comparison of three groups was done by the nonparametric Kruskal-Wallis test. When this was significant, a pairwise Mann-Whitney U-test was performed to identify differences between groups. Correlations were assessed by Spearman's rank correlation coefficients. Data analysis was performed using Prism 5.01 (GraphPad, La Jolla, CA, USA) and Statistical Package for Social Sciences (SPSS, Hong Kong SAR) 17.0. A p-value $<0.05$ was considered significant.

\section{RESULTS}

\section{Clinical characteristics of the study population}

Clinical characteristics of the study population are shown in table 1. Smoking history and all tested lung function parameters were significantly different in the COPD patients when compared to the healthy controls (all $\mathrm{p}<0.0001$ ). Within the COPD group, there was no statistical difference in age or cigarette smoke exposure between moderate, severe and very severe COPD patients.

\section{Characterisation of T-cells}

We quantified the proportions of CD4+ and CD8+ T-cells in peripheral blood (table 2). CD4:CD8 ratios in live PBMC were not significantly different between COPD patients and healthy controls. In addition, we analysed the subpopulations of naïve (CD45RA+CD45RO-) and memory (CD45RA-CD45RO+) CD4+ and CD8+ T-cells. With increasing age the proportions of memory T-cells increased in all study subjects (data not shown); no statistical differences were found between COPD patients and healthy controls (table 2).

Next, we analysed cytokine profiles for IFN- $\gamma$, IL-17A, IL-22, IL$17 \mathrm{~F}, \mathrm{TNF}-\alpha$ and IL-4 upon activation in vitro. In CD4+ T-cells, 
TABLE 1 Subject characteristics

\begin{tabular}{|c|c|c|c|c|c|}
\hline Subjects $n$ & 30 & 10 & 11 & 9 & 10 \\
\hline Sex ratio male/female $n$ & 18/12 & $6 / 4$ & 9/2 & $3 / 6$ & $6 / 4$ \\
\hline Smoking pack-yrs & $43(33-52)^{\#}$ & $50(27-72)$ & $41(27-54)$ & $37(16-57)$ & $0(0-0)^{+}$ \\
\hline Current smoking yes/no $n$ & $9 / 21$ & $5 / 5$ & $4 / 7$ & $0 / 9$ & $0 / 10$ \\
\hline$T L, C O \%$ pred & $58(50-67)^{\#}$ & $70(51-89)$ & $57(49-67)$ & $46(28-63)^{\pi}$ & $112(101-122)^{\bullet}$ \\
\hline KcOc \% pred & $73(63-83)^{\#}$ & $84(63-105)$ & $76(61-92)$ & $55(36-75)^{\pi}$ & $106(100-113)^{\pi /,++}$ \\
\hline
\end{tabular}

Data are presented as mean $(95 \% \mathrm{CI})$, unless otherwise stated. COPD: chronic obstructive pulmonary disease; GOLD: Global Initiative for Chronic Obstructive Lung Disease; FEV1: forced expiratory volume in $1 \mathrm{~s}$; FVC: forced vital capacity; \% pred: \% predicted; $T$ L,CO: diffusing capacity of the lung for carbon monoxide (data available for 27 patients); KcOc: transfer coefficient of the lung for carbon monoxide corrected for the alveolar volume (data available for 27 patients) ${ }^{\#}: p<0.0001$ versus healthy controls; $": p<0.01$ versus all GOLD stages; ${ }^{+}: p<0.001$ versus all GOLD stages; ${ }^{\varsigma}: p<0.01$ versus GOLD IV; ${ }^{f}: p<0.05$ versus GOLD IV; \#\#: $p<0.001$ versus GOLD III; ${ }^{*}: p<0.05$ versus GOLD II; ${ }^{++}: p<0.05$ versus GOLD III and GOLD IV.

cytokine production was almost completely restricted to the CD45RO+ memory T-cell compartment (fig. 1). Although the memory fraction of CD8+ T-cells was the main cytokine producer, also naïve CD8+ cells manifested TNF- $\alpha$ and to a lesser extent IFN- $\gamma$ proportions (fig. 1).

\section{Comparison of cytokine expression between COPD patients and healthy controls}

Proportions of IFN- $\gamma+$ and TNF- $\alpha+$ CD8 + T-cells were significantly increased in COPD patients when compared with healthy controls $(p<0.05$ and $p<0.01$, respectively). Proportions of IL-4+CD8+ cells were low but we observed a trend towards higher proportions of IL-4+ CD8+ T-cells in COPD patients when compared with healthy controls, although significance was not reached. Proportions of IL-17A, IL-22 and IL-17F positive CD8+ cells were negligible and showed no differences between the two groups (fig. 2a).

\begin{tabular}{lcc} 
TABLE 2 & Proportions of lymphocyte subsets \\
& COPD patients & Healthy controls \\
\hline Subjects $\mathbf{n}$ & 30 & 10 \\
CD3+ \% of MNC & $45(36-57)$ & $58(46-67)^{*}$ \\
CD4+ \% of CD3+ & $60(44-68)$ & $63(57-79)$ \\
CD8+ \% of CD3+ & $35(26-44)$ & $28(17-38)$ \\
CD4:CD8 ratio & $1.7(1.2-2.7)$ & $2.3(1.5-4.7)$ \\
CD45RO+ \% of CD3+CD4+ & $48(33-63)$ & $44(24-62)$ \\
CD45RO+ \% of CD3+CD8+ & $19(9.4-26.7)$ & $17(9.5-27.8)$ \\
FoxP3+ Treg \% of CD3+CD4+ & $4.3(3.3-5.6)$ & $3.8(2.8-4.4)$ \\
\hline
\end{tabular}

Data are presented as median (interquartile range), unless otherwise stated. Lymphocyte subsets are presented as proportions of viable cells. COPD: chronic obstructive pulmonary disease; MNC: mononuclear cells; Treg: regulatory T-cell. *: $p<0.05$ versus COPD patients.
Proportions of CD4+ T-cells positive for IL-17A, IL-17F and IL22 were higher than proportions of CD8+ T-cells positive for these cytokines. In contrast to the cytokine production by circulating CD8+ T-cells, proportions of IFN- $\gamma+$ and TNF- $\alpha+$ CD4+ T-cells were not increased in COPD patients, when compared with healthy subjects (fig. 2b). Proportions of CD4+ T-cells expressing the most prominent Th17 cytokines, IL-17A and IL-22 did not show significant differences between COPD patients and healthy controls. Although they comprise a small fraction of circulating CD4+ T-cells, IL-17F+ T-cells were increased in COPD patients, compared with healthy controls (fig. 2b).

\section{Correlations between cytokine expression and lung function parameters}

IFN- $\gamma$ and TNF- $\alpha$ proportions by CD8+ T-cells were higher in COPD patients when compared to healthy subjects. To examine a possible correlation with bronchus obstruction, we examined the differences within the COPD population with different stages of disease. For both cytokines there was a significant correlation present, patients in GOLD stage IV had lower proportions of IFN- $\gamma+\mathrm{CD} 8+$ and TNF- $\alpha+\mathrm{CD} 8+$ T-cells when compared with stage II patients (fig. 3). Also a reduced diffusing capacity was significantly correlated with lower proportions of IFN- $\gamma+\mathrm{CD} 8+$ and TNF- $\alpha+\mathrm{CD} 8+\mathrm{T}$-cells (fig. $4 \mathrm{a}$ and $b$ ). The low but detectable proportions of IL-17A, IL-17F, IL-22 and IL- 4 by CD8+ T-cells did not correlate with either bronchus obstruction (FEV1 or GOLD stage) or lung diffusing capacity $(T \mathrm{~L}, \mathrm{CO})$.

Although, apart from IL-17F, proportions of cytokines produced by CD4+ T-cells did not differ between COPD patients and healthy subjects, we correlated cytokine production with lung function parameters within the COPD patient group. None of the cytokines tested showed a significant correlation with either FEV1 or diffusing capacity except for IL-17A. A reduced diffusing capacity in COPD patients was significantly correlated with lower proportions of IL-17A-expressing CD4+ T-cells. This correlation was present for both TL,CO and KCOc (fig. 4c). 

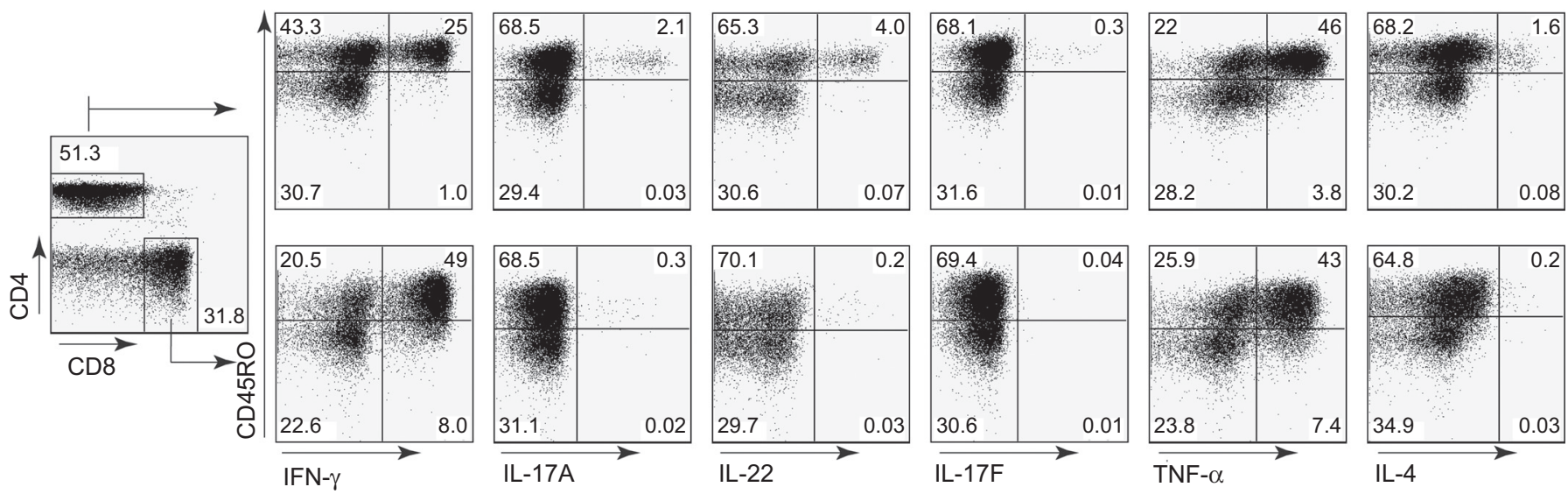

FIGURE 1. Flow cytometric characterisation of T-cells. Peripheral blood mononuclear cell suspensions were stained with monoclonal antibodies specific for CD3, CD4, CD8, CD45RO and the indicated cytokines. Live CD3+ cells were gated and analysed for CD4/CD8 expression. CD4+ (top panels) and CD8+ (bottom panels) T-cells were analysed for the CD45RO memory T-cell marker and the indicated cytokines. Results are shown as dot plots; percentages of cells within the quadrants are given. IFN: interferon; IL: interleukin; TNF: tumour necrosis factor.

As it is conceivable that regulatory T-cells (Tregs) suppress CD4+ T-cells particularly in more advanced COPD, we analysed the population of circulating Tregs. We found no differences in the proportions of FoxP3+CD4+ Tregs between COPD patients and healthy controls (table 2). Likewise, we did not find differences in IL-2R (CD25) or IL-7R (CD127) expression on Tregs between the two groups. Within the COPD population proportions of Tregs did not correlate with either bronchus obstruction or diffusing capacity (data not shown).

\section{Effects of smoking on cytokine expression}

We also examined the potential effect of current smoking on cytokine profiles in COPD patients. Comparisons of current and past smoker COPD cohorts did not reveal significant differences in cytokine production by CD4+ or CD8+ T-cells.
Only for CD4+ T-cells did we observe a trend in higher proportions of IL-22 in current smokers when compared to past smokers (data not shown). Since it was demonstrated that the vast majority of IL-22 producing cells in PBMC were CD4+ T-cells with the CD45RO memory phenotype [21], we performed a subanalysis for IL-22 in the CD45RO+ memory Tcell compartment. Interestingly, CD45RO+CD4+ T-cells of currently smoking COPD patients showed increased proportions of IL-22 when compared to COPD patients that have quit smoking (fig. 5).

\section{DISCUSSION}

In the present study we determined the cytokine profiles of circulating T-cells in patients with stable COPD and correlated these with GOLD classification, lung function parameters and

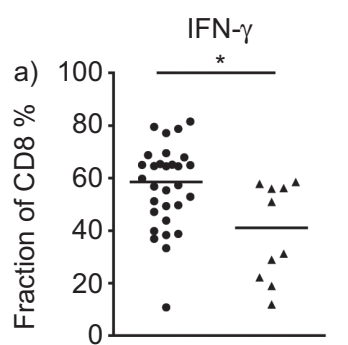

IFN- $\gamma$

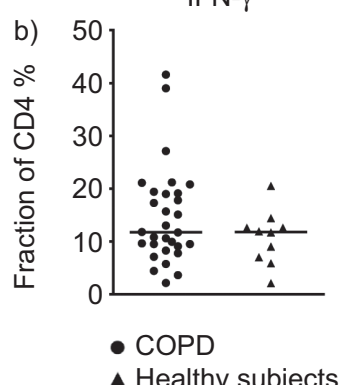

IL-17A

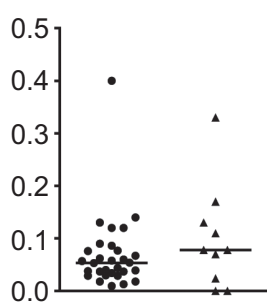

IL-17A

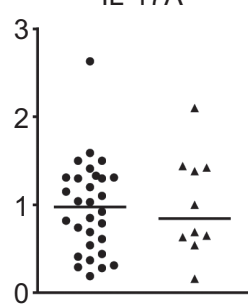

IL-22

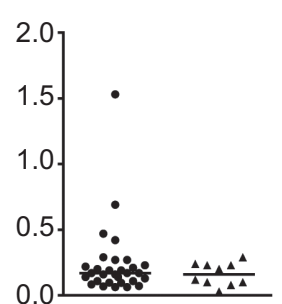

IL-22

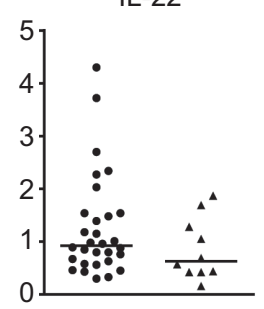

IL-17F

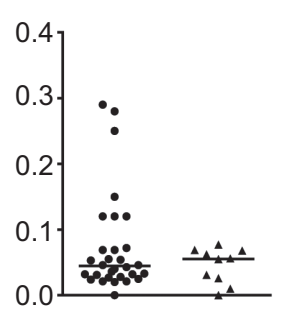

IL-17F

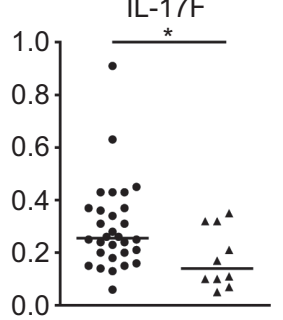

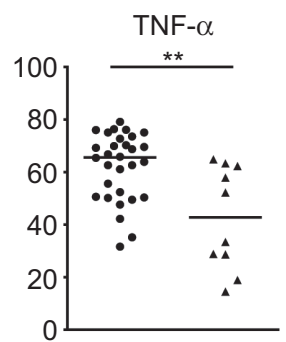

TNF- $\alpha$

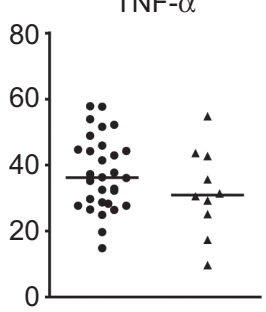

IL-4

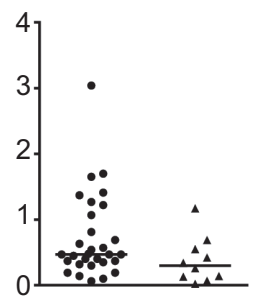

IL-4

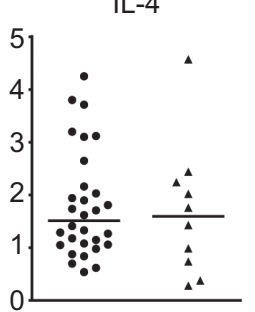

FIGURE 2. Cytokine expression by CD8+ and CD4+ T-cells. a) Proportions of total CD8+ T-cells positive for the indicated cytokines in chronic obstructive pulmonary disease (COPD) patients versus healthy controls. b) Proportions of total CD4+ T-cells positive for the indicated cytokines in COPD patients versus healthy controls. Data are presented as mean \pm SEM. IFN: interferon; IL: interleukin; TNF: tumour necrosis factor. ${ }^{*}: p<0.05 ;{ }^{* *}: p<0.01$. 

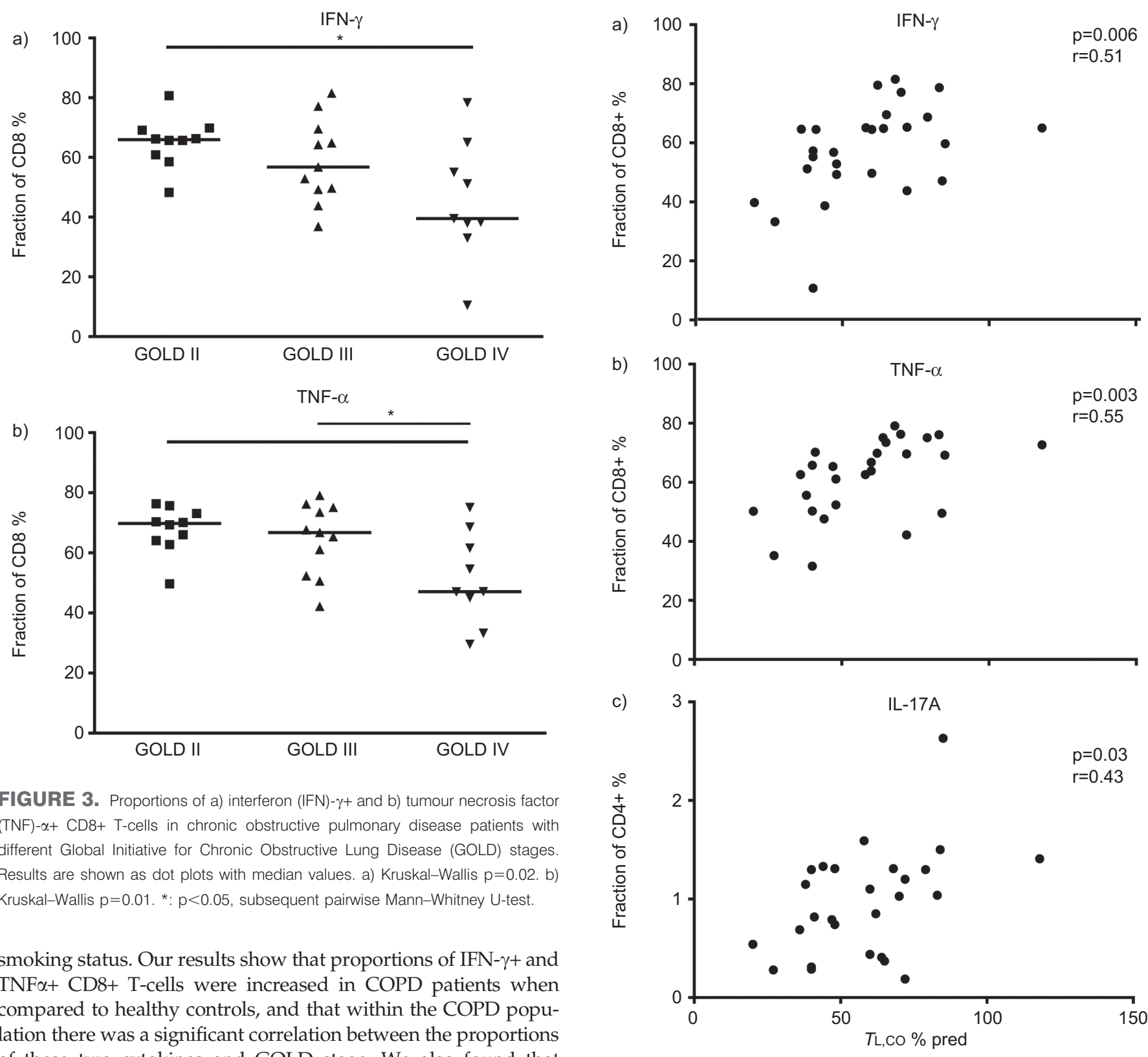

FIGURE 3. Proportions of a) interferon (IFN)- $\gamma+$ and b) tumour necrosis factor (TNF)- $\alpha+$ CD8+ T-cells in chronic obstructive pulmonary disease patients with different Global Initiative for Chronic Obstructive Lung Disease (GOLD) stages. Results are shown as dot plots with median values. a) Kruskal-Wallis $p=0.02$. b) Kruskal-Wallis $p=0.01 .{ }^{*}: p<0.05$, subsequent pairwise Mann-Whitney U-test

smoking status. Our results show that proportions of IFN- $\gamma+$ and TNF $\alpha+$ CD8+ T-cells were increased in COPD patients when compared to healthy controls, and that within the COPD population there was a significant correlation between the proportions of these two cytokines and GOLD stage. We also found that proportions of CD4+ T-cells positive for IL-17A and IL-22 cells were much higher than proportions of CD8+ T-cells positive for these cytokines. COPD patients with more severely reduced diffusing capacity had lower proportions of IL-17A+CD4+ Tcells. Moreover, we show that within the COPD patient population the proportions of IL22+CD4+ memory T-cells are higher in current smokers when compared with past smokers.

Many studies in COPD have focused on the role of the immune system in the lungs $[3,4]$. T-lymphocytes are shown to be extensively present in the alveolar walls of COPD patients and the extent of intrapulmonary lymphocytic infiltrations is correlated with disease severity [2, 4, 10, 22]. It has long been established that CD8+ cells play a major role in the pathogenesis of COPD and that these cells are abundantly present in the airways of patients [3, 4, 10]. It has also been shown that increased COPD disease severity is characterised by an increased cytotoxic potential or cytokine production capacity

FIGURE 4. Correlation of cytokine proportions by CD8+ and CD4+ T-cells with diffusing capacity. Correlations of proportions of a) interferon (IFN)- $\gamma+$ and b) tumour necrosis factor (TNF)- $\alpha+$ CD8+ T-cells with diffusing capacity of the lung for carbon monoxide ( $T \mathrm{~L}, \mathrm{CO} ; \mathrm{n}=27)$. c) Correlations of proportions of interleukin (IL)$17 \mathrm{~A}+\mathrm{CD} 4+\mathrm{T}$-cells with $T \mathrm{~L}, \mathrm{CO}(\mathrm{n}=27) . \%$ pred: \% predicted.

of CD8+ T-cells in the lung [4, 8, 10]. Besides the local inflammation, it is now clear that also systemic inflammation is of great importance in the pathogenesis of COPD [23]. The intensity of this systemic inflammation increases during exacerbations of COPD [22]. However, in contrast with the pulmonary inflammatory component, only little is known about the characteristics and functions of circulating lymphocytes in COPD, and reported findings are often conflicting. In particular, reports on proportions of IFN- $\gamma+$ CD8+ T-cells are confusing. Our finding of increased proportions of IFN- $\gamma+\mathrm{CD} 8+\mathrm{T}$-cells in the circulation of COPD patients is in line with a number of other studies, 


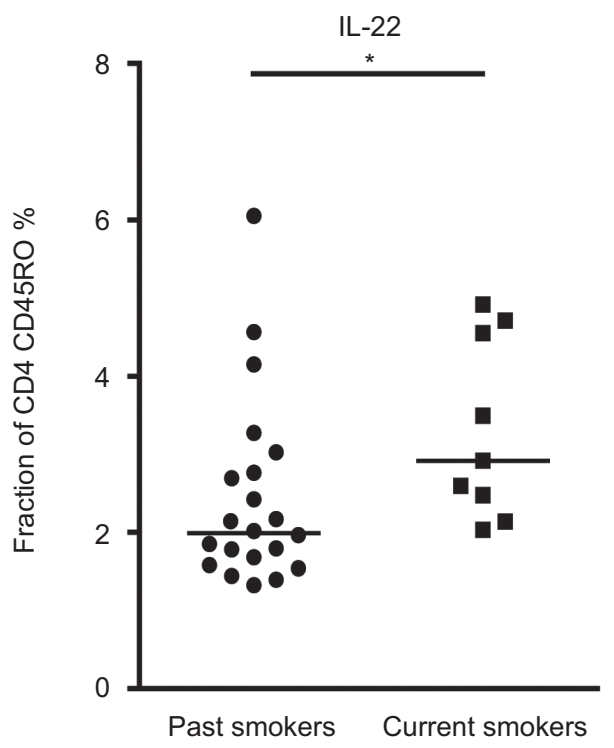

FIGURE 5. The effect of smoking status on interleukin (IL)-22 expression by CD4+ memory T-cells. Proportions of memory CD4+ T-cells positive IL-22 in former smoking versus current smoking chronic obstructive pulmonary disease patients. Results are shown as dot plots with median values. *: $p<0.05$.

including a large study in COPD patients with varying disease severity [24-26]. Moreover, this result is consistent with several reports investigating local inflammation in the lung showing increased IFN- $\gamma$ production by CD8+ T-cells $[5,6]$. In contrast to our finding, REYES et al. [27] reported that CD8+ T-cells from COPD patients produced IFN- $\gamma$ less frequently compared to control subjects. Two other studies could not detect differences in IFN- $\gamma$ production by circulating CD8+ T-cells of COPD patients compared with specimens from healthy subjects [7, 28]. All of these studies were conducted in stable COPD patients; however, disease severity differed greatly.

TNF- $\alpha$ is a potent inflammatory cytokine that enhances neutrophil chemotaxis and migration by inducing the expression of the chemokine CXCL8 (IL-8), which is essential for neutrophil recruitment in COPD. Elevated levels of TNF- $\alpha$ and soluble TNF- $\alpha$ receptors in the serum and sputum of COPD patients suggest an important role for TNF- $\alpha$ in this disease [29]. Yet, reports on TNF- $\alpha$ production by T-cells in the circulation of COPD patients remain limited and conflicting. Our finding of increased TNF- $\alpha$ expression by CD8+ T-cells is supported by HODGE et al. [24]; however, another group reported a decrease in COPD patients when compared with healthy controls [28]. Several studies have attempted to correlate lung function parameters to IFN- $\gamma$ and TNF- $\alpha$ production by T-cells in the circulation [26, 28, 30], but the results are still ambiguous. In our cohort, patients with more severe disease had lower proportions of both IFN- $\gamma+\mathrm{CD} 8+$ and TNF- $\alpha+C D 8+$ T-cells when compared to patients with mild disease. To the best of our knowledge we are the first to report this correlation in proportions of cytokine positive CD8+ Tcells and GOLD stage in the circulation of COPD patients. Our finding does not necessarily imply that progression of COPD to GOLD IV stage results in low proportions of IFN- $\gamma$ or TNF- $\alpha$ expressing circulating CD8+ T-cells or, conversely, that patients with relatively low numbers of IFN- $\gamma$ or TNF- $\alpha$ expressing circulating CD8+ T-cells have an increased susceptibility to progress to GOLD stage IV. In any case, our findings show the importance of analysing the peripheral compartment in the context of COPD heterogeneity [31].

The role of circulating CD4+ cells in the pathogenesis of COPD is not well known. We demonstrated that circulating memory (CD45RO+), but not naïve (CD45RO-) CD4+ T-cells had the capacity to produce cytokines including those of the IL-17 family, upon short stimulation in vitro. For none of the cytokines tested, except for IL-17F, we detected differences in the proportions of cytokine-expressing CD4+ T-cells between the total COPD patient group and healthy controls. Similar to studies on cytokine production by CD8+ T-cells, reports on CD4+ T-cells are conflicting. ZHU et al. [26] found no differences in the cytokine profiles of CD4+ T-cells; however, the group of MAJORI et al. [7] reported an increased percentage of CD4+IFN- $\gamma+\mathrm{T}$-cells in the circulation of COPD patients. To our knowledge, this is the first study to determine systemic Th17 cytokines in COPD. Th17 cells produce pro-inflammatory cytokines, such as IL-17A and IL-22, which can recruit neutrophils to the site of inflammation. Although proportions of the major Th17 cytokines produced by CD4+ memory T-cells did not differ between patients and healthy controls, a correlation with lung function was found within the COPD population. More advanced COPD patients with severely reduced diffusing capacity had lower proportions of IL17A+CD4+ memory T-cells. This could imply that with worsening of emphysema the capacity of IL-17A production by circulating CD4+ memory T-cells is reduced. DI STEFANO et al. [11] investigated the role of Th17 cells in the bronchial mucosa of patients with COPD. In this study there was a significant increase of the number of IL-17A+ cells in the bronchial mucosa of mild/moderate and severe COPD patients compared to control nonsmokers. They suggested that Th17-related cytokines might be involved in the activation of T-cells and endothelial cells reported in patients with COPD. In addition, another study performed by DoE et al. [12] reported an increase in IL-17A+ cells in the submucosa of COPD patients compared with nonsmoking control subjects as well as an increase in sputum IL-17A levels in COPD compared with asthmatic subjects. Further studies, in which peripheral blood and lung tissue are analysed in parallel, should help to elucidate the relationship between Th17 cells in the two compartments in the context of COPD.

Previous studies showed that high doses of ICS may affect the immune system, e.g. by suppressing T-cell function [26]. In our study, all COPD patients received ICS, which minimises confounding by these agents. Although we did not have a control group of COPD patients not using ICS, we verified that our results were not influenced by the dosage of ICS. No correlations were found between ICS doses and FEV1, TL,CO, T-lymphocyte counts or cytokine production, except for proportions of IL17F+CD4+ T-cells, which showed that a higher dose of ICS correlated with lower IL-17F proportions $(\mathrm{p}<0.05$; data not shown).

We have also demonstrated a significant correlation of current cigarette smoking on IL-22 proportions by memory T-helper cells in COPD patients, but not on any of the other cytokines tested. 
Interestingly, cigarette smoke contains many chemicals, including dioxins, which have various toxic effects, most of which are mediated by the aryl hydrocarbon receptor (AHR) [32]. AHR, also called the dioxin receptor, was recently shown to specifically control IL-22 production [33]. It is therefore conceivable that IL-22 production is promoted by direct effects on T-cells of AHR agonists present in cigarette smoke. This is supported by recent findings in the mouse [34] and in humans [35], demonstrating that addition of cigarette smoke extract to T-cells undergoing Th17 differentiation augments the percentage of IL-22 producing cells via an AHR-dependent mechanism. Future studies should also examine a group of smokers with normal lung function to see if these results can be confirmed in this control group.

In our study, the diagnosis of airway obstruction was made based on a FEV1/FVC $<0.70$ as proposed by the GOLD committee in 2001 [19]. However, more recently the GOLD committee recognised that using a fixed value may lead to potential overdiagnosis in the elderly and promoted the use of the lower limit of normal (LLN) instead of a fixed criterion [36]. We verified that there was no misclassification of patients in our study, in all COPD patients in our cohort FEV1/FVC ratio was below the LLN.

We recognise that the observational nature of our study and the relatively small cohort sizes are important limitations to our study. Studies in larger cohorts would be valuable to confirm our results. Another limitation in our study is the observation that patient characteristics revealed an age difference between the COPD patients and the healthy controls (table 1). However, proportions of lymphocyte subpopulations did not differ significantly between these two groups.

In conclusion, our study provides a comprehensive analysis of circulating CD4+ and CD8+ lymphocyte cytokine patterns in stable COPD, which is important since the role of systemic inflammation in the pathogenesis of COPD is not yet clear. Our main findings were that in the peripheral blood of COPD patients the proportions of IFN- $\gamma$ and TNF- $\alpha$ producing CD8+ T-cells were increased when compared with healthy controls and that this was most evident in patients with less severe disease. These results further support the assumption that a systemic inflammation is present in patients with stable COPD. Furthermore, we show a new and interesting correlation between smoking and the proportions of IL-22 expressing CD4+ memory T-cells in peripheral blood. Future studies should also focus on the parallels in systemic and local airway cytokine production in COPD.

\section{STATEMENT OF INTEREST}

None declared.

\section{ACKNOWLEDGEMENTS}

We thank B. van den Blink for critically reviewing the manuscript (Erasmus MC Rotterdam, Rotterdam, the Netherlands).

\section{REFERENCES}

1 Mannino DM. COPD: epidemiology, prevalence, morbidity and mortality, and disease heterogeneity. Chest 2002; 121: Suppl. 5, 121S-126S.
2 Hogg JC. Pathophysiology of airflow limitation in chronic obstructive pulmonary disease. Lancet 2004; 364: 709-721.

3 Hogg JC, Chu F, Utokaparch S, et al. The nature of small-airway obstruction in chronic obstructive pulmonary disease. $N$ Engl J Med 2004; 350: 2645-2653.

4 Barnes PJ. Immunology of asthma and chronic obstructive pulmonary disease. Nature Rev 2008; 8: 183-192.

5 Saetta M, Di Stefano A, Turato G, et al. CD8+ T-lymphocytes in peripheral airways of smokers with chronic obstructive pulmonary disease. Am J Respir Crit Care Med 1998; 157: 822-826.

6 Freeman CM, Curtis JL, Chensue SW. CC chemokine receptor 5 and CXC chemokine receptor 6 expression by lung CD8+ cells correlates with chronic obstructive pulmonary disease severity. Am J Pathol 2007; 171: 767-776.

7 Majori M, Corradi M, Caminati A, et al. Predominant TH1 cytokine pattern in peripheral blood from subjects with chronic obstructive pulmonary disease. J Allergy Clin Immunol 1999; 103: 458-462.

8 Freeman CM, Han MK, Martinez FJ, et al. Cytotoxic potential of lung CD8+ T cells increases with chronic obstructive pulmonary disease severity and with in vitro stimulation by IL-18 or IL-15. J Immunol 2010; 184: 6504-6513.

9 Barczyk A, Pierzchala W, Kon OM, et al. Cytokine production by bronchoalveolar lavage $\mathrm{T}$ lymphocytes in chronic obstructive pulmonary disease. J Allergy Clin Immun 2006; 117: 1484-1492.

10 Curtis JL, Freeman CM, Hogg JC. The immunopathogenesis of chronic obstructive pulmonary disease: insights from recent research. Proc Am Thorac Soc 2007; 4: 512-521.

11 Di Stefano A, Caramori G, Gnemmi I, et al. T helper type 17-related cytokine expression is increased in the bronchial mucosa of stable chronic obstructive pulmonary disease patients. Clin Exp Immunol 2009; 157: 316-324.

12 Doe C, Bafadhel M, Siddiqui S, et al. Expression of the Th17associated cytokines interleukin (IL)-17A and F in asthma and chronic obstructive pulmonary disease. Chest 2010; 138: 1140-1147.

13 Traves SL, Donnelly LE. Th17 cells in airway diseases. Curr Mol Med 2008; 8: 416-426.

14 Agusti AG, Noguera A, Sauleda J, et al. Systemic effects of chronic obstructive pulmonary disease. Eur Respir J 2003; 21: 347-360.

15 Eid AA, Ionescu AA, Nixon LS, et al. Inflammatory response and body composition in chronic obstructive pulmonary disease. Am J Respir Crit Care Med 2001; 164: 1414-1418.

16 Vernooy JH, Kucukaycan M, Jacobs JA, et al. Local and systemic inflammation in patients with chronic obstructive pulmonary disease: soluble tumor necrosis factor receptors are increased in sputum. Am J Respir Crit Care Med 2002; 166: 1218-1224.

17 National Society for Respiratory Physicians. Guideline for diagnoses and antimicrobial treatment of recurrent lower respiratory tract infections. Alphen aan den Rijn, Van Zuiden communications BV, 2005.

18 Fabbri L, Pauwels RA, Hurd SS, et al. Global Strategy for the Diagnosis, Management, and Prevention of Chronic Obstructive Pulmonary Disease: GOLD Executive Summary updated 2003. COPD 2004; 1: 105-141.

19 Pauwels RA, Buist AS, Calverley PM, et al. Global strategy for the diagnosis, management, and prevention of chronic obstructive pulmonary disease. NHLBI/WHO Global Initiative for Chronic Obstructive Lung Disease (GOLD) Workshop summary. Am J Respir Crit Care Med 2001; 163: 1256-1276.

20 Miller MR, Hankinson J, Brusasco V, et al. Standardisation of spirometry. Eur Respir J 2005; 26: 319-338.

21 Liu Y, Yang B, Zhou M, et al. Memory IL-22-producing CD4+ T cells specific for Candida albicans are present in humans. Eur I Immunol 2009; 39: 1472-1479.

22 Hurst JR, Perera WR, Wilkinson TM, et al. Systemic and upper and lower airway inflammation at exacerbation of chronic obstructive pulmonary disease. Am J Respir Crit Care Med 2006; 173: 71-78. 
23 Agusti A. Systemic effects of chronic obstructive pulmonary disease: what we know and what we don't know (but should). Proc Am Thorac Soc 2007; 4: 522-525.

24 Hodge G, Nairn J, Holmes M, et al. Increased intracellular T helper 1 proinflammatory cytokine production in peripheral blood, bronchoalveolar lavage and intraepithelial $\mathrm{T}$ cells of COPD subjects. Clin Exp Immunol 2007; 150: 22-29.

25 Shirai T, Suda T, Inui N, et al. Correlation between peripheral blood T-cell profiles and clinical and inflammatory parameters in stable COPD. Allergol Int 2010; 59: 75-82.

26 Zhu X, Gadgil AS, Givelber R, et al. Peripheral T cell functions correlate with the severity of chronic obstructive pulmonary disease. J Immunol 2009; 182: 3270-3277.

27 Reyes E, Prieto A, de la Hera A, et al. Treatment with AM3 restores defective T-cell function in COPD patients. Chest 2006; 129: 527-535.

28 Barcelo B, Pons J, Fuster A, et al. Intracellular cytokine profile of T lymphocytes in patients with chronic obstructive pulmonary disease. Clin Exp Immunol 2006; 145: 474-479.

29 Oudijk EJ, Lammers JW, Koenderman L. Systemic inflammation in chronic obstructive pulmonary disease. Eur Respir J 2003; 22: Suppl. 46, 5-13.
30 Shirai $\mathrm{T}$, Inui $\mathrm{N}$, Suda $\mathrm{T}$, et al. Correlation between peripheral blood T-cell profiles and airway inflammation in atopic asthma. J Allergy Clin Immun 2006; 118: 622-626.

31 Silverman EK, Vestbo J, Agusti A, et al. Opportunities and Challenges in the Genetics of COPD 2010: An International COPD Genetics Conference Report. COPD 2011; 8: 121-135.

32 Kitamura M, Kasai A. Cigarette smoke as a trigger for the dioxin receptor-mediated signaling pathway. Cancer Lett 2007; 252: 184-194.

33 Trifari S, Kaplan CD, Tran EH, et al. Identification of a human helper $\mathrm{T}$ cell population that has abundant production of interleukin 22 and is distinct from $\mathrm{T}_{\mathrm{H}}-17, \mathrm{~T}_{\mathrm{H}} 1$ and $\mathrm{T}_{\mathrm{H}} 2$ cells. Nat Immunol 2009; 10: 864-871.

34 Chen K, Pociask DA, McAleer JP, et al. IL-17RA is required for CCL2 expression, macrophage recruitment, and emphysema in response to cigarette smoke. PLoS One 2011; 6: e20333.

35 Torii K, Saito C, Furuhashi T, et al. Tobacco smoke is related to Th17 generation with clinical implications for psoriasis patients. Exp Dermatol 2011; 20: 371-373.

36 Rabe KF, Hurd S, Anzueto A, et al. Global strategy for the diagnosis, management, and prevention of chronic obstructive pulmonary disease: GOLD executive summary. Am J Respir Crit Care Med 2007; 176: 532-555. 